See discussions, stats, and author profiles for this publication at: https://www.researchgate.net/publication/342331710

\title{
Breastfeeding and Complementary Feeding Patterns in Namibia
}

Article in Africa Journal of Nursing and Midwifery · December 2019

DOI: 10.25159/2520-5293/4537

\section{CITATIONS}

0

2 authors:

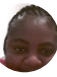

Nelago Indongo

University of Namibia

13 PUBLICATIONS 52 CITATIONS

SEE PROFILE

Some of the authors of this publication are also working on these related projects:

Breastfeeding View project
2 PUBLICATIONS 3 CITATIONS

SEE PROFILE 


\title{
Breastfeeding and Complementary Feeding Patterns in Namibia
}

\author{
Nelago Indongo \\ https://orcid.org/0000-0003-1954-4713 \\ University of Namibia \\ nkanime@unam.na
}

\author{
Klemens Mutorwa \\ https://orcid.org/0000-0002-6713-6610 \\ University of Namibia \\ kmutorwa@unam.na
}

\begin{abstract}
Breastfeeding and complementary feeding practices have significant implications for the child health status. In Namibia, the Maternal and Child Health programme has teamed up with the WHO and UNICEF to improve feeding practices of infants and young children. The main objective of this paper is to examine practices of breastfeeding and complementary feeding among mothers with infants and young children aged 0-24 months. The paper focuses on examining the period of breastfeeding cessation by mothers and the time of the introduction of complementary foods. Information was obtained from 9176 mothers of 16237 infants and young children aged 0-24 months interviewed during the 2013 Namibia Demographic and Health Survey. A survival analysis was used to explore the effects of different variables on the time course of breastfeeding. Although breastfeeding initiation is quite high, most mothers do not continue to breastfeed to 24 months. Among children aged between 0 and 24 months, only 28.2 per cent were still breastfed, and continued breastfeeding is lowly practiced with only 6.1 per cent of children between 20 and 24 months still breastfed. A significant proportion of infants were introduced to solid foods before the recommended age of six months with 31.3 per cent given some solid foods. Developing a breastfeeding culture that involves increasing the duration of maternity leave for working mothers should be considered.
\end{abstract}

Keywords: breastfeeding; children; complementary feeding; infants; Namibia; survival analysis

\section{UNISA $\cong$}




\section{Introduction and Background Information}

The feeding of infants and young children is critical for child health and survival. Based on well-established evidence, the World Health Organization (WHO) and the United Nations International Children's Emergency Fund (UNICEF) recommend that mothers put newborns to the breast within one hour of birth, breastfeed infants exclusively for the first six months and continue to breastfeed for two years and beyond, together with nutritionally adequate, safe and age-appropriate responsive feeding of solid, semi-solid and soft foods starting in the sixth month (Cai, Wardlaw, and Brown 2012,1). Breastfeeding is recognised as the best feeding option for children because of its nutritive, protective, psychological and economic value. The importance of breastfeeding and complementary feeding was underscored by the 2002 World Health Assembly resolution 545.25 on "Global strategy for infants and young children feeding" which re-emphasised the importance of exclusive breastfeeding for six months while promoting the timely introduction of adequate, safe and appropriate complementary feeding together with continued breastfeeding for two years and beyond (Ndiokwelu et al. 2014, 6).

The complementary feeding period is defined as the period during which breast milk must be complemented by other foods of sufficient quantity and quality to cover infants' nutritional needs. The complementary feeding period starts from six to at least 24 months as this is the most critical period for infants' growth and development. The WHO (2008) report indicated that the nutritional inadequacy of the complementary diet, both in quantity and quality, and the undermining effects of infections on the nutritional status of the child remain major challenges affecting infants and young children in the world today. Improving the quality of complementary food has been cited as one of the most cost-effective strategies for improving health, and reducing morbidity and mortality of young children. Nearly one third of child deaths could be prevented by optimal complementary feeding practices (Tariku 2016).

There is strong evidence that the promotion of appropriate complementary feeding practices reduces the incidence of stunting and leads to better health and growth outcomes (Kassa et al. 2016). The decline in the duration of breastfeeding is an inevitable consequence of the modernisation process (Veile and Kramer 2015). Modernisation entails a rapid abandonment of traditional approaches to child-rearing, and the adoption of modern practices including the use of modern health services and the use of supplementary foods for infants in favour of breastfeeding. Veile and Kramer (2015) reported that urbanisation is usually associated with lower incidences and a shorter duration of breastfeeding. The adoption of bottle-feeding in the urban areas is widespread both because it is considered more modern and convenient, and also because there are few breastfeeding role models for urban women to emulate.

The findings of various literature reviews show that the duration of breastfeeding depends on a large number of factors (Akter and Rahman 2010; Ndiokwelu et al. 
2014; Robert et al. 2014). One of the key determinants of the decline in breastfeeding is the increasing level of education among women; a factor which plays a role in the adoption of modern ideas and which usually leads to the abandonment of traditional practices regarding childcare (Ndiokwelu et al. 2014, 14; Robert et al. 2014, 3). Older mothers, multiparity, a higher social class, and the duration of the maternity leave are associated with longer breastfeeding durations (Robert et al. 2014). On the other hand, certain supplementary foods and drinks may be considered culturally important and can affect the timing of the weaning process. Previous research findings (in Ethiopia) on factors associated with appropriate complementary feeding practices show high maternal and paternal education, better household wealth, exposure to the media, adequate antenatal and postnatal contacts, child age, and low parity as determining factors for appropriate complementary feeding (Kassa et al. 2016).

Namibia has made some good progress towards reducing malnutrition of children under five years. The 2013 Namibia Demographic and Health Survey (NDHS) shows a reduction in key anthropometric indicators; stunting (low height for age) has reduced from 29 to 24 per cent, wasting (low weight for height) from 8 to 6 per cent, and underweight (low weight for age) from 17 to 13 per cent between 2006 and 2013 (MHSS and ICF International 2014). While this is an improvement, the rate of reduction in undernutrition is slow. Stunting is a chronic form of undernutrition that is caused by multiple factors, including but not limited to, sub-optimal breastfeeding and complementary feeding practices, poor sanitation and hygiene, the low education level of caregivers, poverty, food insecurity, repeated exposure to infections and disease, and weak health and social welfare systems.

The Government of the Republic of Namibia through its Ministry of Health and Social Services (MHSS) has adopted a primary healthcare strategy in the delivery of health services to the Namibian population immediately after independence. One of the components of the primary healthcare programmes is maternal- and childcare including family planning, immunisations and promotion of breastfeeding and nutrition. Various global instruments as well as national laws and policies have guided the implementation of infant feeding. These include the international code of marketing of breast milk substitutes, the baby-friendly hospital initiative and the babyand-mother-friendly initiatives through the implementation of the ten steps to successful breastfeeding.

In Namibia, the Baby-and-Mother-Friendly Initiative (BMFI) was launched in 1992 (MHSS 1992). The initiative aims to promote, support and protect breastfeeding practices and is adopted from the global Baby-Friendly Hospital Initiative (BFHI). The BMFI guidelines were developed and health workers were trained on breastfeeding management and promotion. Since 1996, 35 state and state-subsidised missionary hospitals were declared baby-and-mother-friendly according to the international criteria of the Ten Steps to Successful Breastfeeding (MHSS 2012). According to the 2006/7 NDHS only 23.9 per cent of children under the age of six 
were exclusively breastfed (MHSS and Macro 2008). This proportion gradually increased to 48.5 per cent in 2013 (MHSS and ICF International 2014). The practice of exclusive breastfeeding in Namibia at the age of 0-1 months was recorded at 72 per cent, 52.7 per cent at 2-3 months, and 26.8 per cent among $4-5$ months old infants. Although the BFHI is in place it only promotes exclusive breastfeeding in the health facility but does not sustain it at community level. In reality many mothers are unable to practice exclusive breastfeeding as advocated (Dhandapany et al. 2008) and there is a paucity of scientific data on reasons why exclusive breastfeeding is not practiced optimally.

\section{Statement of the Research Problem}

The aspect of breastfeeding and complementary feeding patterns has been widely studied in many countries in the region and beyond. However, the pattern and factors associated with and influencing the practices differ by country (Disha et al. 2012; Kassa et al. 2016; Khanal, Sauer, and Zhao 2013). There is a dearth of information on the pattern of breastfeeding and complementary feeding in Namibia, and hence difficult to compare with other countries in the region. Information from the NDHS also indicates that very few mothers achieve the breastfeeding duration they would like to achieve because of various circumstances. This paper therefore assesses the feeding practices and duration of breastfeeding of infants aged 0-24 months as well as factors associated with these practices so as to develop appropriate solutions and possibly strengthen existing programmes in order to achieve longer breastfeeding durations.

\section{Purpose and Objectives}

The purpose of the paper is to examine breastfeeding durations and complementary feeding practices among mothers of infants aged 0-24 months in Namibia, as well as to ascertain the period of breastfeeding cessation by mothers and the time of introduction of complementary foods.

The main objective of the paper is to investigate patterns of breastfeeding and complementary feeding of infants aged 0-24 months in Namibia. Specifically, the paper aims to

- identify factors influencing breastfeeding practices, and

- examine factors associated with the introduction of complementary foods.

\section{Research Methodology}

The data used for analysis have been extracted from the 2013 NDHS. The survey covered 9176 women of reproductive age and a sub-sample 16237 infants aged 0-24 months was used to investigate patterns of breastfeeding and complementary feeding practices. The NDHS is a retrospective cross-sectional survey that provides maternity 
histories completed up to the time of survey. Some variables used in the analysis were recoded into categories of interest. For example, a variable was created to include all children who were given any liquid (other than breast milk). Similarly, a variable was created to include all children who were given any solid food. Descriptive statistics were computed indicating the frequency and averages of variables of interest. Crosstabulations were also run to examine the association between variables. A chi-squared test was used to explain the significance of the relationship. A five per cent level of significance was used.

It is expected that the period between the start and the end of breastfeeding would vary significantly among women. The start time is when breastfeeding starts, i.e. at the birth of a child, and the event of interest is the end of breastfeeding. Thus the women reporting continuation of breastfeeding were regarded as censored cases. The presence of censored data makes conventional statistical techniques inappropriate. Hence, survival analysis has been used in the analysis of breastfeeding cessation where "Time" was the months of breastfeeding and "Status" was whether the mother is still breastfeeding or stopped breastfeeding. This is a method of estimating time to event data in the presence of censored cases. The survival analysis technique takes into consideration both complete and incomplete segments of histories in the breastfeeding analysis. The survival curve was constructed to show the overall pattern and duration of breastfeeding. The analysis was performed in SPSS version 24 .

\section{Analysis}

\section{Description of Variables}

Information from a total of 16237 infants aged 0-24 months was analysed. The average age of the infants was 9.5 months and on average their mothers reported that they breastfed for 8.6 months. The average age of mothers was 35 years and they had an average parity of 3.9 children. The sample consisted of 45.8 per cent infants from an urban area and the majority of children were delivered in a health facility (85.6\%). Only 13.8 per cent of the infants were delivered at home. About 60 per cent of the mothers had at least a secondary education and 48.1 per cent were employed. Attendance of antenatal care was universal and reported by 95 per cent of the mothers. About 89 per cent of the mothers also reported that they had attended prenatal care with nurses compared to only 14.8 per cent who attended prenatal care with doctors. Table 1 presents the mean or percentages of variables examined. 
Table 1: Distribution of means or percentages of variables

\begin{tabular}{|l|l|}
\hline Variable & Mean \\
\hline Mother's age & 35 \\
\hline Months of breastfeeding & 8.6 \\
\hline Age of infant & 9.5 \\
\hline $\begin{array}{l}\text { Total number of children ever } \\
\text { born }\end{array}$ & 3.9 \\
\hline & $\mathbf{n}=\mathbf{1 6} 237(\%)$ \\
\hline Place of residence & \\
\hline Urban & $7442(45.8)$ \\
\hline Rural & $8795(54.2)$ \\
\hline Respondents' education & \\
\hline No education & $1769(10.9)$ \\
\hline Primary education & $4757(29.3)$ \\
\hline Secondary or higher & $9711(59.8)$ \\
\hline Wealth status & \\
\hline Poor & $6973(42.9)$ \\
\hline Rich & $9264(57.1)$ \\
\hline Employed & $7764(48.1)$ \\
\hline Married & $9163(56.4)$ \\
\hline Attend antenatal care* & $2885(95.3)$ \\
\hline Place of delivery: home* & $664(13.8)$ \\
\hline Place of delivery: hospital* & $4115(85.6)$ \\
\hline Prenatal care doctor* & $566(14.8)$ \\
\hline Prenatal care nurse* & $3398(88.6)$ \\
\hline Given other liquids* & $3918(81.6)$ \\
\hline Given solid foods* & $(68.3)$ \\
\hline
\end{tabular}

*Results based on responses received

\section{Breastfeeding Patterns}

Infant feeding indicators were estimated according to the key indicators described by the WHO in 1991 and 2007. These indicators include the timely first suckling rate (the proportion of infants less than 12 months of age who first suckled within 1 hour after birth), the ever breastfed rate (the proportion of infants less than 12 months of age who were ever breastfed), the current breastfeeding rate (the proportion of infants less than 24 months of age who are currently breastfed), the continued breastfeeding rate (1 year) (the proportion of infants 12 to 15 months of age who are breastfed), the continued breastfeeding rate (2 years) (the proportion of infants 20 to 23 months of age who are breastfed), the full breastfeeding rate (the proportion of infants less than 6 months of age who are fed breast milk alone or predominantly breast milk with no additional food based fluids other than fruit juice and sugar water), the bottle-feeding rate (the proportion of infants less than 12 months of age who received any food or drink from a bottle in the previous 24 hours), the timely complementary feeding rate (the proportion of infants 6 to 9 months of age who received complementary foods in 
addition to breast milk in the previous 24 hours), and the median duration of any breastfeeding (the age [in months] when $50 \%$ of children are no longer breastfed).

Infants aged 6-23 months are at a greater risk to suffer from undernutrition (Udoh and Amodu 2016). It is during this period that a child is expected to be introduced to solid foods as primary source of nutrition in accordance with the WHO and UNICEF (2003). When introduced to solid foods, the child may suffer from indigestion, infection, insufficient food or a combination of these. This can result in childhood undernutrition and thus complementary feeding practice is a significant factor that determines the nutritional status of children.

The results show that about 77 per cent of infants were put to breast within one hour after birth. Although breastfeeding initiation is quite high, most mothers do not continue to breastfeed to 24 months. Among infants aged between 0 and 24 months, only 28.2 per cent were still breastfed. Continued breastfeeding is lowly practiced with only 6.1 per cent of infants between 20 and 24 months still breastfed. The results indicate that the proportion of infants below 12 months of age who received any food or drink from a bottle in the previous 24 hours was 18.6 per cent (see Table 2).

Table 2: Breastfeeding indicators among infants 0 to 24 months of age

\begin{tabular}{|l|l|}
\hline Indicator & Percentage \\
\hline Timely put to breast $^{\mathrm{a}}$ & 76.5 \\
\hline Ever breastfed rate $^{\mathrm{a}}$ & 95.7 \\
\hline Current breastfeeding $^{\mathrm{b}}$ & 28.2 \\
\hline Bottle-feeding rate $^{\mathrm{a}}$ & 18.6 \\
\hline
\end{tabular}

${ }^{a}$ infants $<12$ months

${ }^{b}$ infants $0-24$ months

Table 3 shows that there is a significant association between the breastfeeding status and education level of the mother, her employment status, age, prenatal care status, wealth status and place of residence. It also shows the relationship between the bottlefeeding status and a number of socio-economic and demographic characteristics. A chi-squared test is used to test the significance of the association between variables. Results were tested at a five per cent significance level. A p-value of less than 0.05 indicates a significant association between the variables at five per cent while a pvalue less than 0.01 indicates a highly significant association between the variables at one per cent. Breastfeeding decreases with the increasing level of education of the mother. Only 19.6 per cent of infants aged 0-24 months whose mothers had a higher level of education were currently breastfed. A lower proportion of employed mothers (23.5\%) were also currently breastfeeding compared to 33.6 per cent of the unemployed. The results also show that current breastfeeding is low among mothers with a high wealth status and those living in urban areas compared to the poor and those in rural areas (see Table 3). 
Bottle-feeding is highly practiced by mothers with a high level of education (35.9\%) compared to mothers with no education $(6.5 \%)$ or a primary level of education $(11.8 \%)$. The practice of bottle-feeding is also high among employed mothers (24.1\%), mothers with a high wealth status $(24.4 \%)$, and mothers who live in urban areas (25.6\%). A high proportion of infants who feed on bottles are also given other liquids and they are fed on solid foods. Mothers who have given birth to multiples, i.e. twins (28\%), are also likely to use bottle-feeding compared to those with a single birth $(19.3 \%)$. It is important to note that mothers who attend prenatal care practice the use of bottle-feeding less $(21 \%)$ compared to those who do not attend prenatal care $(34.2 \%)$.

\section{Complementary Feeding Practices}

The results indicated that 85.3 per cent of infants aged between 6 and 9 months were introduced to some complementary feeding. Of these, 83 per cent were given liquids other than breast milk and 68 per cent were given solid foods. The solid food mostly introduced among children aged 6-9 months are grains (bread, noodles, etc.), followed by meat $(9 \%)$. Other solid foods were introduced among a lower proportion of children (see Figure 1). Almost 90\% of mothers in urban areas introduced complementary feeding to their infants aged between 6-9 months compared to only 83.4 per cent who did so in rural areas. Similarly, employed mothers and mothers of high wealth status showed a high likelihood of introducing complementary feeding. It is, however, best practice that infants aged between 6 and 9 months are introduced to some complementary feeding. Hence, the proportion is expected to be high, even reaching 100 per cent. It is also noticed that some infants aged between 0 and 6 months were already introduced to complementary feeding. The results show that 35.3 per cent of infants aged 0-6 months were given water, 7.1 per cent were given fruit juice, 7 per cent were given baby formula, and 5.1 per cent were given fresh milk. The results indicated that infants (0-6 months) in urban areas are likely to be given fruit juice (26.3\%) compared to 8 per cent of infants in rural areas who were given fruit juice. They are also likely to be given baby formula. This pattern is consistent for children of mothers with high levels of education and those who are employed. A significant proportion of infants (0-6 months) in the study were also introduced to solid foods before the recommended age of six months with 31.3 per cent given some solid food. 
Table 3: Percentage distribution of current breastfeeding and bottle-feeding by socioeconomic, health and demographic characteristics

\begin{tabular}{|l|l|l|}
\hline Characteristic & $\begin{array}{l}\text { Current breastfeeding } \\
\%(n=4590)\end{array}$ & $\begin{array}{l}\text { Bottle-feeding }^{b} \\
\%(n=4549)\end{array}$ \\
\hline Education & $* *$ & $* *$ \\
\hline No education & $32.0(123)$ & $6.5(25)$ \\
\hline Primary education & $32.7(352)$ & $11.8(127)$ \\
\hline Secondary education & $28.7(835)$ & $22.9(6550)$ \\
\hline Higher education & $19.6(43)$ & $35.9(80)$ \\
\hline Employment status & $* *$ & $* *$ \\
\hline Not employed & $33.6(908)$ & $16.4(440)$ \\
\hline Employed & $23.5(437)$ & $24.1(442)$ \\
\hline Age of mother & $* *$ & $* *$ \\
\hline $15-19$ & $58.0(174)$ & $25.1(73)$ \\
\hline $20-24$ & $32.1(362)$ & $19.3(211)$ \\
\hline $25-29$ & $27.2(331)$ & $18.2(215)$ \\
\hline $30-34$ & $26.0(231)$ & $21.6(195)$ \\
\hline $35-39$ & $25.3(168)$ & $19.6(131)$ \\
\hline $40-44$ & $23.5(72)$ & $15.4(50)$ \\
\hline $45-49$ & $17.2(15)$ & $13.8(12)$ \\
\hline Birth status & $*$ & $*$ \\
\hline Singleton & $29.8(1337)$ & $19.3(857)$ \\
\hline Multiple & $14.8(16)$ & $28.0(30)$ \\
\hline Prenatal care & $*$ & $*$ \\
\hline No prenatal care by nurse & $34.4(142)$ & $34.2(145)$ \\
\hline Prenatal care by nurse & $36.9(1208)$ & $21.0(678)$ \\
\hline Wealth status & $* *$ & $* *$ \\
\hline Poor & $32.9(663)$ & $13.2(265)$ \\
\hline Rich & $26.8(690)$ & $24.4(622)$ \\
\hline Place of residence & $* *$ & $* *$ \\
\hline Urban & $25.5(524)$ & $25.6(522)$ \\
\hline Rural & $32.7(829)$ & $14.5(365)$ \\
\hline Given liquids & & $*$ \\
\hline No & & $10.4(50)$ \\
\hline Yes & & $31.7(629)$ \\
\hline Given solid foods & & $* *$ \\
\hline No & & $31.4(525)$ \\
\hline Yes & & \\
\hline & & \\
\hline
\end{tabular}

${ }^{\mathrm{a}}$ Infants $0-24$ months; ${ }^{\mathrm{b}}$ infants $<12$ months

Chi-squared analysis significance level $* \mathrm{p}<0.05 * * \mathrm{p}<0.01$ 


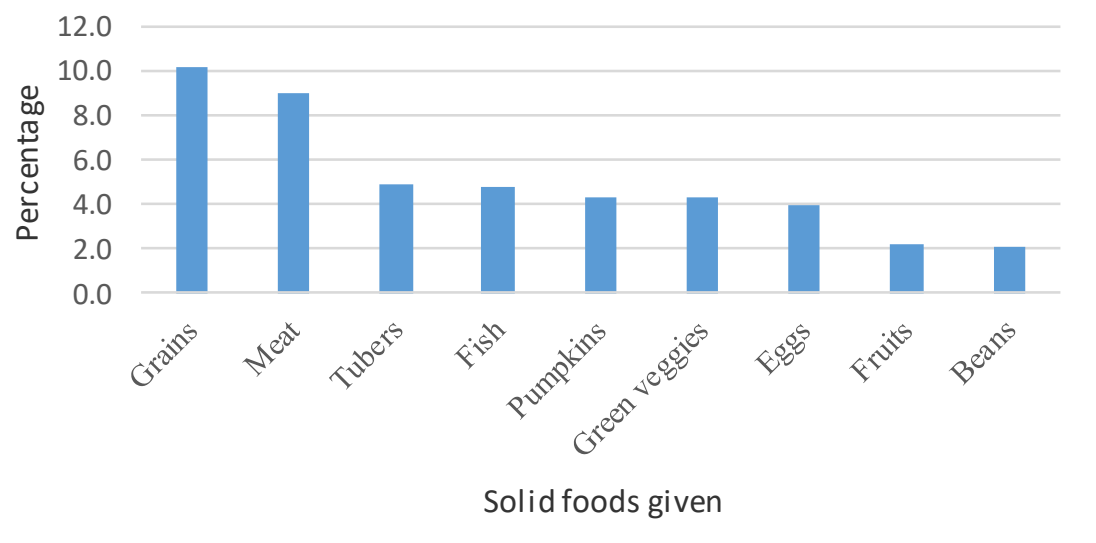

Figure 1: Infants aged 6-9 months by type of solid foods given

The study assessed the relationship between the duration of breastfeeding and the introduction of solid foods (see Figure 2). The results show that breastfeeding decreases as infants get older and are introduced to solid foods. This shows that the probability of breastfeeding decreases as infants are given solid foods, indicating that they are more likely to stop being breastfed as the months of breastfeeding increases. However, the median survival time of breastfeeding is 12 months, implying that some mothers who introduced solid foods continues to breastfeed their children to the age of 1 year.

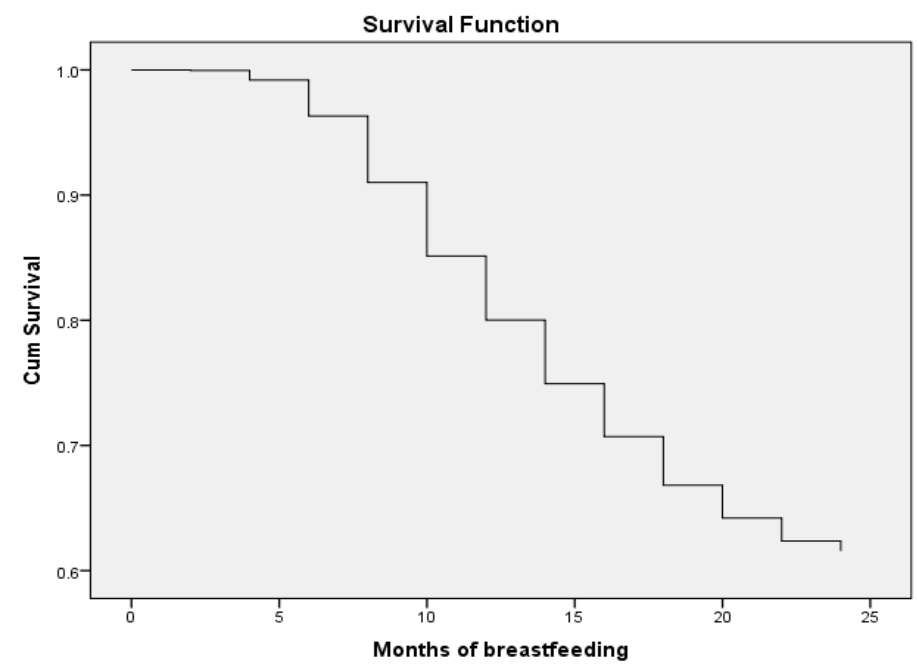

Figure 2: Survival curve for duration of breastfeeding 
Results show that during the first three months of birth only women who are employed introduced solid foods. The results further indicate that employed mothers practice mixed feeding (solid foods and breastfeeding) compared to mothers who are not employed. The study investigated the association between the duration of breastfeeding and the introduction of solid foods among mothers with different levels of wealth status. Most women with a rich wealth status are likely to be employed and likely to have high levels of education, thus a similar pattern was observed, starting solid foods as early within three months compared to poor women who started solid feeding after three months. A survival analysis of the duration of breastfeeding and the introduction of solid foods by birth status was performed. The results show that mothers with multiple births give solid foods earlier than mothers with single births during the first three months of breastfeeding. After the fifth month, the survival curve for mothers with a single birth drops rapidly compared to those with multiple births, which almost flatten to a level plateau suggesting that these mothers are giving solid foods to their infants more than the mothers with a single birth.

\section{Discussion of Research Results}

The WHO (2008) recommends exclusive breastfeeding for six months and advised that complementary feeding must be delayed until six months if the child is born healthy. Udoh and Amodu (2016) reported that the early introduction to solid food is a risk factor for infection and early cessation of breastfeeding. The results from this study show that Namibia does not fully comply with the WHO recommendation of exclusive breastfeeding. The study shows that 35.3 per cent of infants were given water and some were introduced to various types of solid foods between the ages 0 and 6 months. There is a conception that mothers feel that their babies get thirsty if not given anything to drink beside breast milk (Buskens, Jaffe, and Mkhatshwa 2007). These findings concur with other studies where it has been found that water and sugar water are offered even to newborns and thin porridge is offered to the infants as young as two months (Katepa-Bwalya et al. 2015).

Research shows that the early introduction of complementary foods and other liquids puts the child at a high risk of bacteria and other infections that may compromise the child's health (Fewtrell et al. 2017). Thus a child may have had a good start to life with breastfeeding and later on this is compromised and may translate into frequent ill health and malnutrition. Research (Fewtrell et al. 2017; Tariku 2016) emphasised that the timely introduction of solid foods remains an important factor for healthy infant growth. The premature introduction of complementary food was of great concern in this study as well as in other earlier studies (Radwan 2013; Udoh and Amodu 2016) as many mothers still introduced complementary food before the baby reached the age of six months. The high rate of introducing complementary feeding at 6-9 months is encouraging, however, not surprising since a large percentage of mothers already begun feeding complementary foods to their infants before the age of 6 months. 
As a hygiene concern the practice of feeding infants with feeding bottles has been discouraged by the WHO because of the difficulties in maintaining feeding bottles that are free of pathogens which can cause infections. Literature (Udoh and Amodu 2016) indicated that food restrictions owing to cultural practices, unhygienic practices in bottle-feeding, food handling or preparation and responsive breastfeeding are also issues of concern during the complementary feeding period for the child, which result in high incidences of diarrhoea. However, this study shows that employed mothers, mothers with higher levels of education and high economic status continue to practice bottle-feeding more than those who are unemployed, with lower levels of education and poor economic status. The result are consistent with findings by Power et al. (2015) who reported that mothers in medium- and high-income groups introduced other foods earlier compared to those in lower income groups, i.e. 1-2 months in the medium-income group and 5-6 months in the high-income group. Other researchers (Calnen 2010; Smith, Downs, and O'Connell 2001) also argued that owing to the short duration of the maternity leave, working mothers introduce bottle-feeding as soon as they go back to work even when their initial desire was to exclusively breastfeed for six months. A study conducted in the USA reported that the positive effect of maternity leave is substantial. The results from such study indicated that the longer the maternity leave, the longer the duration of breastfeeding (Calnen 2010).

The study further indicated that mothers with multiple births, employed mothers and mothers with wealthy economic status are the most vulnerable group who are at risk of not breastfeeding and introducing solid foods before the recommended age of six months. Proper advice and breastfeeding management are required to increase the awareness of breastfeeding benefits and to ensure that the problems mothers face during breastfeeding do not lead to the cessation of breastfeeding (Radwan 2013). Calnen (2010) reported that breastfeeding initiation is not significantly affected by the employment status. Although employed mothers initiate breastfeeding, Calnen (2010) found that the duration of breastfeeding is significantly longer for non-working mothers than for working mothers, mainly influenced by a short maternity leave. Therefore, a longer paid maternity leave policy of six months might go a long way in helping to eliminate this disparity.

The results also indicate that the age of the mother, the place of residence and the prenatal attendance by a nurse or midwife are factors that are significantly associated with breastfeeding. The association of continuous breastfeeding among mothers who attend prenatal care is an indication of commitment of nurses emphasising or promoting breastfeeding during prenatal care consultations. It was further found that children whose mothers were from rural areas were more likely to breastfeed for a longer time period even while giving solid foods as well as the mothers who had prenatal attendance by a nurse or a midwife. Akter and Rahman (2010) in their study also found that at each point of duration, urban women have a lower probability of continuing to breastfeed relative to rural women. 


\section{Conclusion}

The study concluded that the pattern of breastfeeding among infants and young children is encouraging in Namibia but yet to fully comply with WHO guidelines. The findings indicated that the prevalence of exclusive breastfeeding is still low due to mothers who introduce complementary feeding including solid foods earlier than recommended. The study also concluded that although the breastfeeding rate is high and most infants are put to breast timely, infants are not exclusively breastfed and continuous breastfeeding after one year significantly decreases. Breastfeeding is only high during the first three months and is interrupted with the introduction of supplementary foods from the fourth month. The education level of the mother, her employment status, her wealth status and living in urban area were found to negatively influence breastfeeding practices as well as to be associated with the early introduction of complementary feeding. The attendance of prenatal care was positively associated with a longer duration of breastfeeding and low use of bottle-feeding. Improved knowledge among mothers about infant feeding is needed and this should come from health professionals providing consistent information. Furthermore, a nutritional education intervention, promoting exclusive breastfeeding and the health benefits associated with it, highlighting the health and food safety risks associated with the early introduction of solid foods (especially iron-rich sources) into the infant diets could help reduce the levels of malnutrition, infant infections and mortality in Namibia.

\section{Recommendations}

The study recommends that programmes that promote breastfeeding concentrate on better training for healthcare professionals involved in prenatal care to encourage mothers to exercise exclusive breastfeeding, to avoid bottle-feeding and to continue breastfeeding for two years. Professional advice that focuses on prenatal maternal knowledge may promote adherence to the breastfeeding guidelines of the WHO. It is further recommended that women be discouraged during pregnancy (antenatal care sessions) from introducing breast-milk substitutes before the recommended age. Developing a breastfeeding culture that involves increasing the duration of maternity leave for working mothers should be considered. This will encourage mothers to exclusively breastfeed their babies. There is also a need for a national communitybased breastfeeding intervention programme as part of a primary public health strategy to decrease health risks and problems among infants and children.

\section{Limitations of the Study}

Like any other studies using secondary data, this study has some limitations. There is the possibility that some of the responses might suffer from memory recall as well as socially desirable responses. The reporting and recall bias is a common problem to the retrospective data used, relying on memory of past events. The cross-sectional nature of the data used for the analysis prevents the study from determining causality. 
However, the use of nationally representative data (the NDHS) lends strength and credibility to the analyses, and the use of the NDHS to identify policy and programme priorities is highly appropriate (Disha et al. 2012; Khanal, Sauer, and Zhao 2013).

\section{Acknowledgements}

We acknowledge the Namibia Ministry of Health and Social Services for providing the data. We also thank the University of Namibia for the time granted to work on this manuscript.

\section{References}

Akter, S., and M. Rahman. 2010. "Duration of Breastfeeding and its Correlates in Bangladesh." Journal of Health Population Nutrition 28 (6): 595-601. https://doi.org/10.3329/jhpn.v28i6.6608.

Buskens, I., A. Jaffe, and H. Mkhatshwa. 2007. "Infant Feeding Practices: Realities and Mind Sets of Mothers in Southern Africa." AIDS Care 19 (9): 1101-9. https://doi.org/10.1080/09540120701336400.

Cai, X., T. Wardlaw, and D. W. Brown. 2012. "Global Trends in Exclusive Breastfeeding." International Breastfeeding Journal 7:12. https://doi.org/10.1186/1746-4358-7-12.

Calnen, G. 2010. "The Impact of Maternity Leave on Breastfeeding Rates.” Breastfeeding Medicine 5 (5): 233-4. https://doi.org/10.1089/bfm.2010.0064.

Dhandapany, G., A. Bethou, A. Arunaginathan, and S. Ananthakrishanan. 2008. “Antenatal Counseling on Breastfeeding - Is it Adequate? A Descriptive Study from Pondicherry, India." International Breastfeeding Journal 3:5. https://doi.org/10.1186/1746-4358-3-5.

Disha, A. D., R. Rawat, A. Subandoro, and P. Menon. 2012. "Infant and Young Child Feeding (IYCF) Practices in Ethiopia and Zambia and Their Association with Child Nutrition: Analysis of Demographic and Health Survey Data." African Journal of Food, Agriculture, Nutrition and Development 12:2.

Fewtrell, M., J. Bronsky, C. Campoy, M. Domellöf, N. Embleton, M. N. Fidler, I. Hojsak, J. M. Hulst, F. Indrio, A. Lapillonne, and C. Molgaard. 2017. "Complementary Feeding: A Position Paper by the European Society for Paediatric Gastroenterology, Hepatology, and Nutrition (ESPGHAN) Committee on Nutrition." Journal of Pediatric Gastroenterology and Nutrition 64 (1): 119-32. https://doi.org/10.1097/MPG.0000000000001454.

Kassa, T., B. Meshesha, Y. Haji, and J. Ebrahim. 2016. “Appropriate Complementary Feeding Practices and Associated Factors among Mothers of Children Age 6-23 Months in Southern Ethiopia 2015." Biomedical Central Pediatrics 16:131. https://doi.org/10.1186/s12887-016-0675-X. 
Katepa-Bwalya, M., V. Mukonka, C. Kankasa, F. Masaninga, F., O. Babaniyi, and S. Siziya. 2015. "Infants and Young Children Feeding Practices and Nutritional Status in Two Districts of Zambia." International Breastfeed Journal 10:5. https://doi.org/10.1186/s13006-015-0033-x.

Khanal, V., K. Sauer, and Y. Zhao. 2013. "Determinants of Complementary Feeding Practices among Nepalese Children Aged 6-23 Months: Findings from Demographic and Health Survey 2011." Biomedical Central Pediatrics 13:131. https://doi.org/10.1186/1471-243113-131.

MHSS (Namibia Ministry of Health and Social Services). 1992. Towards a Baby and Mother Friendly Nation: Guideline for the implementation of the Baby and Mother Friendly Initiative. Windhoek: Namprint.

MHSS (Namibia Ministry of Health and Social Services). 2012. National Guidelines on Infant and Young Child Feeding. Windhoek: Primary HealthCare Directorate.

MHSS (Namibia Ministry of Health and Social Services) and ICF International. 2014. The Namibia Demographic and Health Survey 2013. Windhoek: MHSS and ICF International.

MHSS (Namibia Ministry of Health and Social Services) and Macro (Macro International Inc.). 2008. Namibia Demographic and Health Survey 2006-07. Windhoek: MHSS and Macro.

Ndiokwelu, C. I., A. N. Maduforo, C. A. Amadi, and C. P. Okwy-Nweke. 2014. "Breastfeeding and Complementary Feeding Practices of Mothers of Children (0-24 Months) Attending Infant Welfare Clinic (IWC) at the Institute of Child Health (ICH) University of Nigerian Teaching Hospital (UNTH) Ituku-Ozalla Enugu.” Journal of Biology, Agriculture and Healthcare 4:11.

Power, T. G., S. O. Hughes, L. S. Goodell, S. L. Johnson, J. A. J. Duran, K. Williams, A. D. Beck, and L. A. Frankel. 2015. "Feeding Practices of Low-Income Mothers: How do They Compare to Current Recommendations." International Journal of Behavioral Nutrition and Physical Activity 12:34. https://doi.org/10.1186/s12966-015-0179-3.

Radwan, H. 2013. "Patterns and Determinants of Breastfeeding and Complementary Feeding Practices of Emirati Mothers in the United Arab Emirates." Biomedical Central Public Health 13:171. https://doi.org/10.1186/1471-2458-13-171.

Robert, E., Y. Coppieters, B. Swennen, and M. Dramaix. 2014. "Breastfeeding Duration: A Survival Analysis - Data from a Regional Immunization Survey." Biomedical Research International 2014. https://doi.org/10.1155/2014/529790.

Smith, K., B. Downs, and M. O'Connell. 2001. Current Population Reports: Maternity Leave and Employment Patterns: 1961-1995. Washington: U.S. Census Bureau. 
Tariku, A. 2016. "Effect of Feeding Style on Intake of Complementary Foods Appetite and Nutritional Status of Infants Aged 9-11 Months in West Gojam Ethiopia.” Master's thesis, Addis Ababa University.

Udoh, E. E., and O. K. Amodu. 2016. "Complementary Feeding Practices among Mothers and Nutritional Status of Infants in Akpabuyo Area, Cross River State Nigeria." Springerplus 5 (1): 2073. https://doi.org/10.1186/s40064-016-3751-7.

Veile, A., and K. Kramer. 2015. "Birth and Breastfeeding Dynamics in a Modernising Indigenous Community.” Journal of Human Lactation 31 (1): 145-55. https://doi.org/10.1177/0890334414557177.

WHO (World Health Organization). 1991. Indicators for Assessing Breastfeeding Practices. Geneva: WHO.

WHO (World Health Organization). 2007. "Indicators for Assessing Infant and Young Child Feeding.” In Consensus Meeting, edited by B. Daelmans, 19. Washington: WHO.

WHO (World Health Organization). 2008. "Indicators for Assessing Infant and Young Child Feeding Practices. Part 1.” Definitions, Conclusions of a Consensus Meeting held 68 November 2007 in Washington DC, USA.

WHO and UNICEF. 2003. Global Strategy for Infants and Young Child Feeding. Geneva: WHO. 